\title{
Erratum: Emergent and broken symmetries of atomic self-organization arising from Gouy phase shifts in multimode cavity QED [Phys. Rev. A 99, 053818 (2019)]
}

\author{
Yudan Guo, Varun D. Vaidya, Ronen M. Kroeze, Rhiannon A. Lunney, Benjamin L. Lev, and Jonathan Keeling
}

(Received 17 December 2020; published 5 January 2021)

DOI: 10.1103/PhysRevA.103.019901

A number of small errors appeared in some of the equations in this article. The results presented in the article are not altered. In the following, we note these errors and provide the corrected equations.

(1) In Eqs. (2), (B1), and (B2) a factor of 1/2 was missing in defining the wavefront curvature. The equation should read

$$
\hat{\phi}(\mathbf{r})=\Omega \cos \left(k_{r} x\right)+g_{0} \sum_{\mu, Q} \hat{a}_{\mu, Q} \Xi_{\mu}(\mathbf{r}) \cos \left[k_{r}\left(z+\frac{r^{2}}{2 R(z)}\right)-\theta_{\mu, Q}(z)\right],
$$

in order that the radius of curvature $R(z)$ is consistent with the standard definition $R(z)=z+z_{R}^{2} / z$, which was quoted in the paper. The corrected form of (B1) similarly becomes

$$
\Phi(\mathbf{r}, z) \propto \sum_{\mu} \alpha_{\mu} \Xi_{\mu}\left(\frac{w_{0} \mathbf{r}}{w(z)}\right) \cos \left[k_{r}\left(z+\frac{r^{2}}{2 R(z)}\right)-\Theta_{Q_{0}}-n_{\mu} \theta_{0}(z)\right] \mathcal{S}_{\mu}^{+} .
$$

The corrected form of (B2) is given below.

(2) The unnumbered equation after Eq. (4) had an incorrect sign and missing parentheses in the exponent. This should have read

$$
\Psi(\mathbf{x})=Z\left(z-z_{0}\right) \sqrt{\rho(\mathbf{r})}\left[\psi_{0}+\sqrt{2} \cos \left(k_{r} x\right) \operatorname{Re}\left\{\psi_{1} e^{-i\left(k_{r} z+\delta\right)}\right\}\right] .
$$

(3) Equation (8) had an incorrect numerical prefactor and had implicitly assumed $\psi_{0}$ to be real. The corrected equation should be

$$
\begin{aligned}
i \partial_{t} \psi_{\sigma}= & \int d \mathbf{r}\left(\mu+2 \omega_{r}\right) \rho(\mathbf{r}) \psi_{\sigma}+\frac{1}{2} \int d \mathbf{r} U \psi_{0}^{2} \psi_{\sigma}^{*} \rho(\mathbf{r}) \\
& +\frac{g_{0}^{2} \Omega^{2} N}{4 \Delta_{a}^{2} \Delta_{\mu_{0}, Q_{0}}} \int d \mathbf{r} \int d \mathbf{r}^{\prime} \sum_{\tau=c, s} \operatorname{Re}\left\{\mathcal{D}_{\sigma \tau}\left(\mathbf{r}, \mathbf{r}^{\prime}\right)\right\} \rho(\mathbf{r}) \rho\left(\mathbf{r}^{\prime}\right)\left[\left|\psi_{0}\right|^{2} \psi_{\tau}+\psi_{0}^{2} \psi_{\tau}^{*}\right] .
\end{aligned}
$$

(4) Equation (17) and subsequent symmetrized Green's functions were missing a factor of $1 / 2$. The corrected definition is as follows:

$$
\mathcal{G}^{+}\left(\mathbf{r}, \mathbf{r}^{\prime}, \varphi\right)=\frac{1}{2}\left[\mathcal{G}\left(\mathbf{r}, \mathbf{r}^{\prime}, \varphi\right)+\mathcal{G}\left(\mathbf{r},-\mathbf{r}^{\prime}, \varphi\right)\right] .
$$

This similarly adds a prefactor of $1 / 2$ in Eqs. (22) and (23), and the unnumbered equation after Eq. (39).

(5) In Eqs. (19) and (20), an overall minus sign should be added for the expression of $\alpha_{\mu}$ so that

$$
\alpha_{\mu}=-\frac{\Omega g_{0} N}{2 \sqrt{2} \Delta_{a}} \int d \mathbf{r}^{\prime} \frac{\Xi_{\mu}\left(\mathbf{r}^{\prime}\right)}{\Delta_{\mu}+i \kappa} \rho\left(\mathbf{r}^{\prime}\right) \sum_{\sigma=c, s} \mathcal{O}_{\mu}^{\sigma} \chi_{\sigma},
$$

and, consequently, the first line of Eq. (20) should read

$$
\begin{aligned}
\tilde{\alpha}(\mathbf{r}, z) & \equiv \sum_{\mu} \alpha_{\mu} \Xi_{\mu}(\mathbf{r}) \cos \left(k_{r} z-\Theta_{Q_{0}}-n_{\mu} \theta_{0}\right) \\
& =-\frac{\Omega g_{0} N}{2 \sqrt{2} \Delta_{a}} \int d \mathbf{r}^{\prime} \sum_{\mu, \sigma=c, s} \frac{\Xi_{\mu}(\mathbf{r}) \Xi_{\mu}\left(\mathbf{r}^{\prime}\right)}{\Delta_{\mu}+i \kappa} \cos \left(k_{r} z-\Theta_{Q_{0}}-n_{\mu} \theta_{0}\right) \mathcal{O}_{\mu}^{\sigma} \chi_{\sigma} \rho\left(\mathbf{r}^{\prime}\right) .
\end{aligned}
$$

(6) Throughout the paper, the scale of the transverse coordinate has an implicit dependence on the $z$ coordinate (i.e., the cavity axis). At each location $z$, for quantities involving the cavity transverse field profile $\Xi_{\mu}(\mathbf{r})$, the transverse coordinate $\mathbf{r}$ 
should be evaluated as $\mathbf{r} \rightarrow w_{0} \mathbf{r} / w(z)$, where $w(z)=w_{0} \sqrt{1+z^{2} / z_{R}^{2}}$ is the explicit dependence of cavity waist. This was not previously stated clearly. The implicit dependence was made explicit in Appendix B, but this was treated inconsistently in Eq. (B2). This equation should have read

$$
\Phi(\mathbf{r}, z) \propto \operatorname{Re}\left(\int d \mathbf{r}^{\prime} \rho\left(\mathbf{r}^{\prime}\right) D_{3 D}\left(\frac{w_{0} \mathbf{r}}{w(z)}, \frac{w_{0} \mathbf{r}^{\prime}}{w\left(z_{0}\right)} ; z\right) \exp \left\{i\left[k_{r}\left(z+\frac{r^{2}}{2 R(z)}\right)-\Theta_{Q_{0}}\right]\right\}\right) .
$$

Note that we also corrected the factor of $1 / 2$ in the radius of curvature as described above.

(7) In Eq. (B3), factors of $i$ were missing from the arguments of the Green's function,

$$
\begin{aligned}
4 D_{3 D}\left(\mathbf{r}, \mathbf{r}^{\prime} ; z\right)= & G\left[\mathbf{r}, \mathbf{r}^{\prime},-i \theta_{0}(z)+i \theta_{0}\left(z_{0}\right)\right]+e^{2 i \tau} G\left[\mathbf{r}, \mathbf{r}^{\prime},-i \theta_{0}(z)-i \theta_{0}\left(z_{0}\right)\right] \\
& +G\left[\mathbf{r}, \mathbf{r}^{\prime},-i \theta_{0}(z)+i \theta_{0}\left(z_{0}\right)+i \pi\right]+e^{2 i \tau} G\left[\mathbf{r}, \mathbf{r}^{\prime},-i \theta_{0}(z)-i \theta_{0}\left(z_{0}\right)+i \pi\right] .
\end{aligned}
$$

(8) The forms of Eqs. (B4) and (B5) have an incorrect factor of 2 in front of the cloud width $\sigma_{A}^{2}$. The corrected equations are

$$
\begin{aligned}
\int d \mathbf{r}^{\prime} \rho\left(\mathbf{r}^{\prime}\right) G\left(\mathbf{r}, \mathbf{r}^{\prime},-i \phi\right)= & \frac{w_{0}^{2} e^{-i \phi}}{2 \pi\left(2 \sigma_{A}^{2} \cos \phi-i w_{0}^{2} \sin \phi\right)} \\
& \times \exp \left[-\frac{\mathbf{r}_{0}^{2} \cos \phi-2 \mathbf{r}_{0} \cdot \mathbf{r}+\mathbf{r}^{2}\left(w_{0}^{2} \cos \phi-i 2 \sigma_{A}^{2} \sin \phi\right) / w_{0}^{2}}{2 \sigma_{A}^{2} \cos \phi-i w_{0}^{2} \sin \phi}\right],
\end{aligned}
$$

and

$$
\Phi_{\text {mid }}(\mathbf{r}) \propto \frac{w_{0}^{2}}{4 \pi \sigma_{A}^{2}}\left[\exp \left(\frac{-\left(\mathbf{r}-\mathbf{r}_{0}\right)^{2}}{2 \sigma_{A}^{2}}\right)+\exp \left(\frac{-\left(\mathbf{r}+\mathbf{r}_{0}\right)^{2}}{2 \sigma_{A}^{2}}\right)\right]+\frac{e^{2 i \tau}}{\pi} \exp \left(-\frac{2 \sigma_{A}^{2}|\mathbf{r}|^{2}}{w_{0}^{4}}\right) \cos \left(\frac{2 \mathbf{r} \cdot \mathbf{r}_{0}}{w_{0}^{2}}\right),
$$

where $\Phi_{\text {mid }}$ indicates that this refers to the transverse field profile at the midplane $z=0$ of the cavity. 